\title{
Cientintifica
}

http://revistas.udistrital.edu.co/ojs/index.php/revcie/index

Artículo de INVESTIGACIÓN

\section{La educación energética, una prioridad para el milenio}

\author{
The energy education a priority for the millennium
}

A energia da educação uma prioridade para o milênio

Johnn Édgar Castro ${ }^{1}$

Adriana Patricia Gallego Torres ${ }^{2}$

Fecha de recepción: junio 2014

Fecha de aceptación: noviembre 2014

Para citar este artículo: Castro, J. y Gallego A. (2015). La educación energética, una prioridad para el milenio. Revista Científica, 21, 111-120. Doi: 10.14483/udistrital.jour.RC.2015.21.a11

\section{Resumen}

Este artículo plantea la necesidad de reflexionar y trabajar en el desarrollo de propuestas sobre educación energética, fundamentalmente desde cuatro enfoques: la inevitable dependencia de las sociedades humanas de las fuentes de energía; el ahorro energético; el agotamiento de las fuentes de energía y, por último, las consecuencias sobre el medioambiente.

Palabras Clave: educación energética, energías renovables, didáctica de las ciencias.

\section{Abstract \\ This article raises the need to reflect and work on the development of proposals on energy educa- tion, mainly from four approaches: the first is the in- evitable dependence of human societies of energy}

sources; energy savings; depletion of energy sources and finally the impact on the environment.

Keywords: energy education, renewable energy, science education.

\section{Resumo}

Este artigo levanta a necessidade de reflectir e trabalhar no desenvolvimento de propostas sobre educação para a energia, principalmente a partir de quatro abordagens: a primeira é a inevitável dependência das sociedades humanas de fontes de energia; economia de energia; depleção de fontes de energia e, finalmente, o impacto sobre o meio ambiente.

Palavras-chave: educação para a energia, energias renováveis, educação científica. 


\section{Introducción}

Hoy existe un conjunto de problemáticas y de posibles soluciones relacionadas con la energía que intentan diversificar el modelo energético actual y que buscan suplir las necesidades energéticas de una manera más responsable y adecuada, que no afecte la vida en el planeta. Sin embargo, los problemas persisten y parecen superar las soluciones. Desde esta perspectiva queremos enfocar nuestra investigación, debido principalmente a que estamos convencidos de que la situación energética actual es una realidad de contrastes donde se agudizan los problemas energéticos: por un lado, se hace más evidente el agotamiento de los combustibles de origen fósil y, por otro lado, se agravan los problemas ambientales que generan su explotación, transporte y uso, además de que las diferencias sociales y económicas empeoran la situación por la falta de acceso a las fuentes y las tecnologías energéticas.

En el marco de la educación científica, tecnológica y ambiental se han propuesto y desarrollado formas alternativas de educación; una es la educación energética, la cual ha sido reconocida como una propuesta de carácter planetario a la problemática energética, que se ha venido desarrollando desde hace cuatro décadas en diversos países, cada uno atendiendo a su contexto (Castro Montaña, 2012; Castro Montaña, 2015). Esto ha sido motivado por diferentes causas, las cuales se propone agrupar en cuatro categorías: la primera es la inevitable dependencia de las sociedades humanas de las fuentes de energía; la segunda, el ahorro económico, en tanto que entre menos se consuma energía menos se debe invertir en su producción, especialmente los países importadores de fuentes de energía; la tercera, el inminente agotamiento de las fuentes de energía de origen fósil, haciendo referencia especial al agotamiento del petróleo; y la cuarta, la afectación negativa sobre el ambiente cuando se hace uso inadecuado de la fuentes de energía.
Si bien ellas se relacionan entre sí y se presentan de manera casi simultánea, se han organizado de la anterior manera atendiendo a la importancia que históricamente ha tenido en las sociedades humanas, pues sin duda alguna lo primero que preocupó al ser humano fue conseguir fuentes de energía para suplir sus necesidades energéticas, antes que preocuparse por los costos, el agotamiento o inclusive el daño al ambiente. Aunque los altos costos de las fuentes de energía están asociados a su agotamiento, particularmente en el caso del petróleo, las propuestas en principio se desarrollan para reducir los gastos económicos de generación y producción de energía, más que para crear una conciencia del agotamiento de las fuentes y por ende de las limitaciones energéticas del planeta. Así que, sin duda alguna, guiados por la ahorro de dinero, esto implicó tomar conciencia del agotamiento de los recursos y de las afectaciones negativas del uso inadecuado de la fuentes de energía sobre el ambiente.

\section{La inevitable dependencia de las sociedades humanas de las fuentes de energía}

Las sociedades humanas para poder existir han dependido de los usos que han dado a las diversas fuentes de energía. Al respecto, Postigo (1965) muestra cómo la energía no es un agente descubierto recientemente, sino que las primeras acciones humanas implicaron el uso de la energía: cuando se buscó alimento y vestido o se controló el fuego; pero más allá, el uso de fuentes de energía permitió el desarrollo tecnológico que configuró sociedades distintas. Por ejemplo, la energía humana fue durante mucho tiempo una fuente de energía muy utilizada que permitió el desarrollo de civilizaciones antiguas, como los caldeos, asirios, persas, egipcios, griegos, romanos, chinos, aztecas e incas. Indudablemente de manera individual la energía humana es escasa, pero en masa puede llegar a ser muy importante, además, porque el ser humano cuenta con la capacidad intelectual, esto lo llevó a compensar sus falencias mecánicas, 
aunque a costa de colocar a un grupo significativo de seres humanos en condición esclavitud.

Un avance que permitió en parte reducir el esfuerzo físico del ser humano fue la energía animal, sin tener una fecha exacta de la domesticación de los animales, a partir de las inscripciones de civilizaciones antiguas como los sumerios, hititas y caldeos se estima que la domesticación del caballo se dio alrededor del 5000 antes de Cristo. A lo largo de su historia, el ser humano ha logrado domesticar de manera exitosa un poco más de veinticinco especies de mamíferos, diez de aves y dos de insectos, entre los que se cuenta el gusano de seda y la abeja.

El uso de las fuentes de origen fósil, de acuerdo con Postigo (1965), marcó otro nivel en el desarrollo de las sociedades. Por ejemplo, si bien el carbón mineral fue utilizado como combustible de manera doméstica, en las herrerías y alguna otra industria menor, durante varios siglos y en el siglo XVI se exportaba carbón de Inglaterra a Francia y a los Países Bajos, fue aproximadamente a comienzos del siglo XVIII que se dio inicio a la era del carbón, debido a tres razones: el agotamiento de los bosques, el descubrimiento de que el uso del carbón mineral en los altos hornos aumentaba su rendimiento y el desarrollo y perfeccionamiento de la máquina de vapor por Watt en 1782 .

Posteriormente, el uso del petróleo a gran escala comenzó sustituyendo paulatinamente el carbón mineral en los procesos industriales, pero fue mayor su uso cuando se constituyó como combustible de automóviles y otros medios terrestres. En 1908, cuando Henry Ford construyó el automóvil Ford tipo T, que para 1910 ya circulaban alrededor de unos 200.000, generó una demanda de bencina de petróleo mayor para automóviles que lo solicitado hasta el momento para la industria. Luego, en 1914, en Inglaterra substituyeron el carbón por el petróleo en el funcionamiento de sus buques. Durante la Primera Guerra Mundial se hizo uso de esencia de petróleo en las máquinas acorazadas, aviones y camiones de transporte a un nivel nunca utilizado. En 1937, en Estados Unidos, se inicia la tracción diesel en los ferrocarriles, que substituye al carbón mineral, y en la década de los cuarenta del siglo XX, en ese país y en Europa occidental se generalizó el uso de derivados del petróleo para la calefacción doméstica.

Asimismo, ocurrió con el gas natural: aunque su utilidad, al parecer, se conoció por primera vez en China cuando lo utilizaron para evaporar agua de mar y obtener sal, su uso en alumbrado público se remonta a 1802, en la ciudad de Génova Italia (Camuzzi Gas Pampeana, 2010). Para 1960, los italianos producían aproximadamente 6.248 millones de metros cúbicos de gas natural. Hoy muchos hogares cuentan con el servicio de gas natural para la cocción de alimentos y la calefacción, y muchos automóviles hacen uso del gas natural vehicular.

Aunque parezcan de reciente desarrollo y aplicación, las energías alternativas cuentan con una historia más amplia de lo que se logra suponer. Según Postigo (1965), es altamente probable que la idea de utilizar la energía solar de manera directa corresponda a civilizaciones como la de los egipcios, chinos o griegos, los cuales seguramente la utilizaron para el secado de los productos agrícolas, de las ropas, de materiales de construcción como los ladrillos y la evaporación del agua marina para la obtención de sal. El uso de la energía solar a nivel industrial comenzó en 1872 al norte de Chile, donde se construyó la primera planta desalinizadora del mundo, con el fin de suministrar agua potable a una mina ubicada en la región. Uno de los primeros artefactos que aprovechó la radiación solar directa fue el calentador solar de agua, el cual fue inventado en 1891 por Clarence Kemp, quien lo comercializó bajo el nombre de Climax en la región de California a un precio de 25 dólares (DForce, 2010).

Otra de las energías alternativas de gran desarrollo es la energía eólica, la cual utiliza el viento como fuente de energía. Una de sus primeras aplicaciones fue en el uso de la vela para impulsar embarcaciones, sin embargo, uno de los artefactos que ha permitido el uso del viento como agente 
energético es el molino de viento. Su origen es difícil de determinar, pero se dice que lo inventaron en Asia suroriental, en China e India y que fue llevado a Francia por los primeros cruzados aproximadamente en 1180 y luego se extendió a los Países Bajos. En el 2010, Noruega anunció la construcción de un molino de viento, o dicho de mejor manera, de una turbina eólica con una hélice de 145 metros de diámetro, con una capacidad de producir energía eléctrica con una potencia de 10MW (Embajada de Noruega, 2010).

Otra fuente de energía alternativa es la energía hidráulica, de la cual se estima que su aprovechamiento se remonta a la invención de rueda hidráulica, probablemente desarrollada por el pueblo chino hace más de 4.000 años, quienes la utilizaron y aún la utilizan en espacios rurales, en la elevación de agua para el riego de cultivos. Un adelanto tecnológico que superó la eficiencia de la rueda hidráulica fue la turbina, que aprovecha la energía de caída del agua, la cual entra a la turbina y actúa con todas las paletas de manera simultánea, siempre en el mismo sentido y no como en la rueda hidráulica donde el agua solo actúa con una parte de las paletas de la rueda. Entre las turbinas que han favorecido la construcción de hidroeléctricas están la Kaplan, la Francis y la Pelton.

La energía es uno de los aspectos de mayor relevancia en el funcionamiento de las sociedades contemporáneas, más aún cuando se enfrenta el reto de la supervivencia del ser humano en el planeta en la actualidad y por ende en el futuro. Así lo entiende la Asamblea General de las Naciones Unidas al proclamar el 2012 como Año Internacional de las Energías Sostenibles para Todos. Este organismo reconoce que la energía tiene un profundo efecto en la productividad, la salud, la educación, el cambio climático, la seguridad alimentaria e hídrica y los servicios de comunicación (ONU, 2010; OEI, 2012).

El reconocimiento de lo energético como un aspecto importante ya se venía haciendo evidente en eventos internacionales, como la Cumbre de la Tierra en Río de Janeiro en 1992, donde por medio del Programa 21 se planteó el desarrollo, eficiencia y consumo de la energía como un área a trabajar en la conservación y gestión de los recursos para el desarrollo, particularmente en la protección de la atmósfera, en tanto que se reconoce que la energía es esencial para el desarrollo económico y social y el mejoramiento de la calidad de vida, pero teniendo en cuenta que las formas de utilizar las fuentes de energía deben enmarcarse en el respeto a la atmósfera, la salud humana y el ambiente en su totalidad, además se ve la necesidad de eliminar los obstáculos al aumento del suministro de energía ecológicamente racional, especialmente en los países en desarrollo (ONU, 1992).

La energía tiene un gran valor en la vida cotidiana, en tanto que hablar de energía en la sociedad actual es común ya que dicho concepto se ha ido incorporado de manera gradual en el acervo cultural, como un concepto científico que tiene muchas implicaciones en la vida cotidiana de las personas o inclusive como una mercancía, una entidad de valor económico y social. Se puede decir, como bien lo plantea Arrastía que "conocer sobre energía constituye un elemento esencial para la cultura general e integral de cualquier persona en el siglo XXI" (2007, p. 24). Resulta, entonces, que la energía es un concepto fundamental en la vida del ser humano que debe ser abordado por todos los ciudadanos en el transcurso de su vida.

\section{La necesidad de ahorrar gastos económicos en la generación de energía}

En principio, las propuestas en educación energética se configuraron como una forma de ahorrar dinero, ya que enseñar a las personas a ahorrar energía es una forma de reducir el gasto de capital al reducir el consumo de energía. Al respecto, Pichs (2007) plantea que países importadores de petróleo, como Cuba, Brasil, Chile, Estados Unidos y los de la Unión Europea, frente a los precios elevados de esta fuente de energía tuvieron la necesidad de desarrollar propuestas de educación energética. 
Según Blanco (2006), las políticas energéticas de los países desarrollados dependientes del petróleo, luego de la crisis energética los llevó al planteamiento e incorporación de tres principios básicos: la contención en el consumo, el aumento de la eficiencia energética y el desarrollo de nuevas fuentes de energía, como la nuclear o las energías alternativas, principios que se han operativizado, en parte, en propuestas de educación energética.

Tales propuestas en educación energética, como las de García, Mendoza y Bermejo (2007), asumen una actitud crítica frente a concepciones como que la energía ya no es una forma de suplir necesidades básicas y algunos lujos, sino que es una mercancía que debe venderse produciendo grandes beneficios. En la propuesta de Figueroa et al. (2009) se reconoce que la energía no es en sí misma un bien para el consumo final, sino un bien intermedio para satisfacer otras necesidades en la producción de bienes y servicios. Desde el punto de vista tecnológico y económico, la energía es un recurso natural primario o derivado que permite realizar un trabajo o servir de subsidiario en actividades económicas independientes de la producción de energía. Por su parte, Dias, Mattos y Balestieri (2004) consideran que la energía es fundamental para el desarrollo social, económico y la estabilidad de cualquier país, por lo que su uso racional consiste en un conjunto de acciones que representa la búsqueda del equilibrio entre el binomio disponibilidad-consumo de la relación energía y el estado del ambiente.

El carácter económico de la educación energética ha conllevado a mostrar que es una propuesta económicamente rentable. Por ejemplo, Brasil, según Días, Mattos y Balestieri (2004), y las Comunidades Europeas (2006) muestran que propuestas de educación energética, orientadas a medidas de ahorro de energía, en el marco del Programa Nacional de Conservación de la Electricidad (Procel) permitieron que en 1998 no se generaran 1.560 megavatios, que sumado a las mejoras de la eficiencia en centrales eléctricas, generó un aumentó en la producción en 1,4 TWh, estas dos acciones permitieron que el pueblo brasileño se ahorrara cerca de 3100 millones de dólares de inversión en nuevas centrales eléctricas.

Si bien una educación energética se muestra pertinente en países importadores de petróleo, también lo es en países que lo exportan. Un ejemplo de esto es el caso de Colombia, que exporta petróleo crudo, pero no tiene una capacidad significativa en los procesos de refinación, pues su principal actividad económica es la producción agrícola. Según Pichs (2007), los ingresos adicionales por las exportaciones suelen retornar a los países industrializados a través de canales comerciales y financieros internacionales, más aún cuando lo que se exporta es petróleo crudo, que es más barato, además si se exportan otras cosas como productos agrícolas, manufacturados u otro tipo de productos que dependan del consumo derivados del petróleo se reducirá su ganancia, porque parte de su gasto la está dejando en la producción y transporte del producto. Es decir, entre más costoso sea el petróleo y se siga dependiendo de él, los países categorizados como pobres en el actual modelo económico no seguirán siendo pobres sino más pobres, en tanto que su deuda externa será cada vez más grande. En este punto vale la pena preguntar: ¿será la exportación de petróleo crudo una buena opción económica, social y ambiental para los colombianos?, ¿de qué maneras se posicionan los profesores de ciencia en relación con la producción y exportación de petróleo en Colombia?

\section{El inminente agotamiento de las fuentes de energía no renovables}

El innegable carácter socioeconómico motivó el desarrollo de la educación energética, pero no fue el único argumento que se esgrimió: otro argumento ha sido frenar el inminente agotamiento de las fuentes de energía no renovables. La mayoría de los especialistas en educación energética ((Dias, Mattos y Balestieri, 2004; Blanco, 2006; Domínguez y Pérez, 2010; Figueroa et al., 2009; Pérez, Sánchez, 
Villegas, Sánchez García y Pérez, 2006; Arrastía, 2007; Pichs, 2007; Ávila, Cruz y Núñez, 2010) reconocen que una de las problemáticas asociadas a las fuentes de energía de origen fósil es su agotamiento, debido a que son fuentes no renovables, lo que implica que su disponibilidad es limitada. A esto ha de sumarse que el consumo mundial de energía continuará aumentando en los próximos decenios y la dependencia del petróleo no cederá significativamente (Romero-Barcos, 20066) . El agotamiento será ineludible (Pichs, 2007).

Centrar la mirada en el agotamiento de las fuentes de energía en la educación energética trasciende la preocupación económica, si bien es un buen argumento que favorece el ahorro económico, permite, además, que las personas comprendan que las fuentes naturales son limitadas, que en algún momento se agotaran, más aún si se aumenta su consumo. Uno de los elementos motivadores de la mirada sobre el agotamiento de las fuentes de energía fue la publicación en 1972 del libro Los límites del crecimiento, informe del Club de Roma, donde se ponía de manifiesto que los factores que limitaban el crecimiento en el planeta Tierra eran: la población, producción agrícola, producción industrial, la contaminación y recursos naturales, así como que un incremento de los cuatro primeros implica una reducción dramática del último, lo que Ilevaría al planeta a un punto que no podría soportar (Mayor, 2009). Ya no se trata solamente de cuidar un capital, sino de cuidar el planeta, no agotarlo, utilizar sus fuentes de manera moderada. Poner de presente la preocupación por el agotamiento de las fuentes de energía es reconocer las limitaciones que se tienen para crecer económicamente, crea la necesidad de ser moderados en el consumo.

A pesar de que este problema ha sido central en las propuestas de educación energética desde sus inicios, además de que se reconoce que en teoría trasciende el problema económico, en la práctica lamentablemente lo financiero sigue primando. según la British Petroleum (2012), Estados Unidos, país con una trayectoria reconocida en propuestas de educación energética, en el 2011 tenía una reserva probada de treinta mil millones de barriles de petróleo, diariamente produjo 7.841 .00 millones de barriles y consumió 18.835 .000 barriles, lo que implica que no solamente está agotando sus reservas probadas, sino que además está agotando las de otros países, solamente por mantener su crecimiento económico.

En el caso colombiano no existen propuestas en educación energética reconocidas, además se presenta un panorama preocupante de la relación crecimiento económico-agotamiento del petróleo. Según la British Petroleum (2012), en el 2011, Colombia tenía una reserva probada de dos mil millones de barriles, producía 930.000 barriles diarios y consumía 230.000 barriles diarios; de seguir produciendo de esta manera agotará sus reservas en aproximadamente seis años. Es de tener en cuenta que Colombia exportó en promedio en ese año 630.000 barriles de petróleo diario, lo que representa el $67 \%$ de su producción, lo que implica que más que atender un necesidad energética propia con sus fuentes, lo que hace es negociar en el mercado a pesar de que esto significa a corto plazo un agotamiento del petróleo, lo que obliga a preguntar: cuando se agote el petróleo, ¿qué medidas se tomarán para suplir las necesidades energéticas?, ¿importar petróleo, reemplazarlo con otra fuentes de energía o dejar de suplir las necesidades energéticas? Es evidente que lo que busca Colombia no es reducir el agotamiento del petróleo, sino aumentar su ganancia económica, lo que indica que se requiere una educación energética distinta a las propuestas en países como Estados Unidos, que contribuya efectivamente a mitigar el agotamiento de las fuentes de energía no renovables.

El argumento del agotamiento si bien es válido, no es un argumento transferible a todas las regiones ni a todos los países del mundo de la misma manera, en tanto que los consumos de energía son diferenciados. Para Vilches, Gil, Toscano y Macias (2007), hacer referencia al agotamiento de las fuentes no renovables de energía es obligatorio pero no suficiente, porque a pesar de ser un 
problema reconocido, los países desarrollados siguen teniendo altos niveles de consumo de este tipo de fuentes. Según la British Petroleum (2012), en el 2011 los Estados Unidos consumió el 20,5\% de la producción mundial de petróleo, seguido por China con $11,4 \%$, luego Japón con un 5\%, la India con un 4\%, la Federación Rusa con un 3,4\%, Arabia Saudita con un 3,1\%, Brasil con un 3\%, mientras que Colombia consumió tan solo el $0,3 \%$ de dicha producción.

Las diferencias que se presentan son grandes: si bien la población de los Estados Unidos es siete veces más numerosa que la población colombiana, el consumo de petróleo en los Estados Unidos es setenta y cuatro veces más que en Colombia; de la misma manera, China — con una población treinta veces más grande que la de Colombia - tiene un consumo treinta y nueve veces mayor de petróleo. Lo anterior permite ver que hay un desequilibrio en el consumo, pues aunque todos consumen, no todos lo hacen de la misma manera y en la misma proporción, por lo tanto, la responsabilidad es distinta frente al agotamiento de los recursos. Mientras que para los que hiperconsumen energía, una educación energética debe ser más insistente para tomar conciencia de la responsabilidad que tienen en el agotamiento de fuentes de energía, para los pequeños consumidores, la educación energética se refiere a evitar llegar a niveles de hiperconsumo y contribuir en la formación de un pensamiento crítico y en una acción consecuente que permita exigir a los que hiperconsumen reduzcan su consumo y asuman la responsabilidad de los daños generados para así impedir que los modelos de vida basados en el consumo sigan proliferando en la sociedad.

\section{La afectación negativa sobre el ambiente en el uso inadecuado de la energía}

1. crear en los individuos la conciencia y la mejor compresión de los problemas que afectan al ambiente en el mundo contemporáneo, haciendo uso de los hallazgos de la ciencia y la tecnología, procurando que puedan desempeñar una función productiva con miras a mejorar la vida y proteger el ambiente, prestando la debida atención a los valores éticos;

2. fomentar la elaboración de comportamientos positivos de conducta frente al ambiente y la utilización que la nación le da a sus recursos;

3. crear una nueva perspectiva general donde se reconozca la existencia de una profunda interdependencia en el medio natural y el medio artificial;

4. poner de manifiesto la continuidad permanente que vincula los actos del presente a las consecuencias del fututo, además de la interdependencia entre las comunidades nacionales y la necesaria solidaridad entre todo el género humano.

De manera más específica, Soberats et al. (2005) afirman que los profesores que asumen la educación energética deben enfocar sus esfuerzos en preservar el ambiente favoreciendo un aprendizaje sobre el uso eficaz de los recursos energéticos del mundo en el presente y en el futuro, sin que se deje de satisfacer la demanda creciente de energía de una población en rápido aumento e industrialización.

En Colombia, la realidad energética en relación con los problemas ambientales es de contrastes. Colombia tiene una producción de energía eléctrica basada en un $70 \%$ en hidroeléctricas, lo que conlleva una producción baja de $\mathrm{CO} 2$ y metano que otros países. Según el protocolo de Kioto, Colombia no está obligada a reducir las emisiones de gases de efecto invernadero. Además cuenta con una política en educación ambiental por medio de la cual, a partir del Decreto 1793 de 1994, el Ministerio de Educación Nacional y el Ministerio de Medio Ambiente se unieron para acompañar la inclusión y desarrollo de proyectos ambientales escolares (PRAES) en todas la instituciones de educación formal y promover la educación ambiental no formal e informal; esta política en educación ambiental ha sido valorada por investigadores en 
educación ambiental reconocidos a nivel mundial como Lucie Sauvé (2004) como una política de carácter comunitario.

No obstante, en términos energéticos, por un lado, se ha promovido la ampliación y mantenimiento de termoeléctricas que funcionan con carbón mineral, a pesar del potencial hidroeléctrico del país. En 2011, según la British Petroleum (2012), Colombia figura como el tercer país que más incrementó su producción de carbón en el mundo, sumando siete millones de toneladas equivalentes de petróleo a su producción del 2010, además se muestra que incrementó en un 25,5\% su consumo de carbón entre 2010 y 2011. Por otro lado, se ha promovido la construcción de grandes hidroeléctricas, como la del Quimbo, que ha sido la causa de problemas ambientales, porque su construcción ha implicado la desviación del río Magdalena y la inundación de grandes extensiones de suelo rico en fauna y flora, además de incidir negativamente en la dinámicas sociales y culturales de los habitantes de la región. resulta atendiendo una demanda de energía eléctrica de otros países, haciéndola ver como una opción económica más que una alternativa que busca suplir necesidades energéticas locales. Frente a lo anterior, se hace evidente que la educación ambiental colombiana debe atender los problemas ambientales que generan la explotación y producción de fuentes de energía en el país.

\section{A manera de conclusión}

La educación energética no es una propuesta nueva, sino que tiene su origen en la década de los setenta del siglo XX, cuando tuvieron lugar las crisis energéticas de 1973 y 1979, que se manifestaron más como crisis económicas generadas por la situación comercial del petróleo a nivel mundial, cuando este se hizo más costoso y se tomó conciencia de que no era una fuente energética infinita sino que podría agotarse en corto tiempo. Frente a lo anterior, los países desarrollados dependientes del petróleo introdujeron tres principios básicos en sus políticas energéticas: la contención en el consumo, el aumento de la eficiencia energética y el desarrollo de nuevas fuentes de energía como la nuclear o las alternativas. En el marco de la contención del consumo de energía, la educación energética comenzó a configurarse, y es ahí precisamente donde emerge la primera acepción de esta, asociada a campañas de ahorro de energía.

Las primeras propuestas de educación energética vieron la luz a mediados de la década de los setenta y finales de la década de los ochenta del siglo XX. Al respecto, Glass (1985) asegura que la primera vez que se usó el término educación energética (energy education) como descriptor en ERIC fue en julio de 1978.

Aunque hoy en día la educación energética contempla como acción importante el ahorro de energía, ya sea porque es una opción económica para hacer frente a los elevados precios del petróleo en el mercado internacional, o porque se ha convertido en una opción para hacer frente a los problemas ambientales derivados del uso de la energía, no se reduce a esta recomendación. Actualmente, la educación energética se ve abocada en los países de bajos recursos económicos, también, para hacer frente a la creciente pobreza pues se asume que esta situación está vinculada con la carencia de sistemas adecuados de energía para cubrir necesidades básicas (Pichs, 2007). Al concebirse la educación energética como una opción para contribuir de manera simultánea a reducir el gasto de energía, cuidar el planeta y garantizar la configuración de sistemas energéticos adecuados para cubrir necesidades básicas de los más pobres, se convierte, entonces, en una propuesta educativa que no es exclusiva de algunos países, sino de todo el planeta.

\section{Referencias}

Arrastía, M. y Yanes, E. (2006). El programa de capacitación para cuadros y docentes de la Universidad Pedagógica Enrique José Varona. En J. Mendoza y M. Fernández (Eds.), Educación, 
Enerxía e Desenvolvemento Sostible (pp. 103124). Santiago de Compostela: Universidad Santiago de Compostela.

Arrastía, M. (2007). Educación Energética de respeto ambiental. Premisa para el desarrollo sostenible. En J. Mendoza Rodríguez, M. Fernández Domínguez, y M. Arrastía Ávila (Eds.), Educación enerxética e desenvolvemento sostible (pp. 23-33). Santiago de Compostela: Servizo de Publicacións e Intercambio Científico, Campus Universitario Sur, Santiago de Compostela. Ávila, E., Cruz, M. y Núñez, G. (2010). La educación energética para el desarrollo sostenible: un desafío en el siglo XXI. Observatorio Iberoamericano del Desarrollo Local y la Economía Social - OIDLES, 4(8), 1-10.

Blanco, F. (2006). ¿Estamos fomentando las renovables? Una mirada desde la docencia. En J. Mendoza Rodríguez, y M. Fernández Domínguez (Eds.), Educación, Enerxía e Desenvolvemento Sostible (pp. 137-144). Santiago de Compostela: Universidad de Santiago de Compostela.

Bourne, J. (2010). Derrame de petróleo en el Golfo. Un dilema profundo. National Geographic, 2-31.

British Petroleum. (2012). BP Statistical Review of World Energy June 2012. Disponible en http:// www.bp.com/

Camuzzi Gas Pampeana. (2010). Historia del Gas Natural. [En línea]. Recuperado de http:// www.camuzzigas.com/perfil-corporativo-historia-del-gas-natural. Consultado el 1 de febrero de 2011.

Castro, J. (2012). Argumentos para una educación energética en Colombia: Entre lo planetario, lo contextual y la no transferencia. Educyt, Extraordinario, 41-67.

Castro, J. (2015). La educación energética en las universidades públicas de Bogotá: una mirada a las licenciaturas en Física. (Tesis Doctoral). Doctorado Interinstitucional en Educación, Universidad Distrital Francisco José de Caldas, Bogotá.

Comunidades Europeas. (2006). Educación energética. Enseñar a los futuros consumidores de la energía. [En línea]. Luxemburgo: Ofi- cina de Publicaciones Oficiales de la Comunidades Europeas. Recuperado de http:// www.managenergy.net/download/education2005/05-0001-ES.pdf

DForce. (2010). Energías Renovables. Recuperado el 3 de 2 de 2011, de gstriatum.com: http://www. gstriatum.com/energiasolar/blog/2010/05/26/ quien-invento-el-calentador-solar/

Dias, R., Mattos, C., y Balestieri, J. (2004). Energy education: breaking up the rational energy use barriers. Energy Policy, 31(11), 1339-1347.

Domínguez, Z., y Pérez, N. (2010). Tendencias históricas de la educación energética en la formación de profesionales de la educación en Cuba: implicaciones para el colectivo pedagógico. SIMPOSIO I: Década de la educación para el desarrollo sostenible- Importancia de la educación científica para la cultura energética y medioambiental (pp. 1-14). La Habana: VI Congreso Internacional Didácticas de las ciencias. XI Taller Internacional sobre la Enseñanza de la Física.

Embajada de Noruega. (05 de 04 de 2010). Construyen molino de veinto con tecnología innovadora. Recuperado el 10 de 2 de 2011, de noruega. org.ar: http://www.noruega.org.ar/News_and_ events/Noticias-de-Noruega/Construyen-molino-de-viento-con-tecnologia-innovadora/

Figueroa, J., Villalobos, R., Contreras, C., Latorre, X., Hanh, N., Chaparro, C., . . . Figueroa, R. (2009). Eficiencia energética en la enseñanza media científica humanística. Guía de apoyo docente. Chile: CONAMA Nacional.

Flannery, T. (2006). El clima está en nuestras manos ( $1^{\text {a }}$ ed.). (Trad. D. Alou). New York: Taurus.

García, J., Mendoza, J. y Bermejo, M. (2007). Las centrales hidroeléctricas en Galicia: producción energética e impacto ambiental. En J. Mendoza, M. Fernández y M. Arrastía (Eds.), Educación Enerxética e Desenvolvemento Sostible (pp. 141-158). Santiago de Compostela: Servizo de Publicacións e Intercambio Científico, Campus Universitario Sur, Santiago de Compostela.

Glass, L. (1985). Energy Education. Focus on excellence. Ames, lowa: National Science Foundation. 
Gore, A. (2007). Una verdad incómoda: La crisis planetaria del calentamiento global y cómo afrontarla ( $1^{\text {a }}$ ed.). Madrid: Gedisa.

Instituto Mundial del Carbón. (2005). El carbón como recurso. Una visión general del carbón. Londres: World Coal Institute.

Intergovernmental Panel on Climate Change (IPCC). (2007). Cambio climático 2007: Informe de síntesis. Contribución de los grupos I, II y III al Cuarto Informe de evaluación del Grupo Intergubernamental de Expertos sobre el Cambio Climático. R. K. Pachauri y A. Reinsinger (Eds.) Ginebra, Suiza: IPCC.

Isaza, J. F. y Campos, D. (2007). Cambio climático. Glaciaciones y calentamiento global. Bogotá: Fundación Universidad de Bogotá Jorge Tadeo Lozano.

Lovelock, J. (2007). La venganza de la Tierra: La teoría de Gaia y el futuro de la humanidad ( $1^{\mathrm{a}} \mathrm{ed}$.). (Trad. M. García Puig). Barcelona: Planeta.

Mayor, F. (2009). Los límites del crecimiento. Tribuna Libre, pp. 10-16.

Organización de Estados Iberoamericanos (OEI). (2012). 2012 Año Internacional de la Energía Sostenible para Todos. Recuperado de http:// www.oei.es/divulgacioncientifica/spip.php?article380. Consultado el 13 de enero de 2012.

Organización de las Naciones Unidas (ONU). (2011). Resolución 65/151. Año Internacional de la Energía Sostenible. Asamblea General Naciones Unidas.

Organización de las Naciones Unidas (ONU). (1992). Cumbre para la Tierra. Programa 21. Programa de Acción de las Naciones Unidas de Río. Río de Janeiro: Naciones Unidas.

Pérez, R. (2006). Retos de la educación energética en el siglo XXI. En J. Mendoza y M. Fernández (Eds.), Educación, Enerxía e Desenvolvementos Sostible (pp. 515-522). Santiago de Compostela: Universidad de Santiago de Compostela.

Pérez, O., Sánchez, N., Villegas, A., Sánchez García, T. y Pérez, E. (2006). Educación energética de los ciudadanos, ¿un capricho o una nece- sidad? En J. Mendoza y M. Fernández (Eds.), Educación, enerxía e desenvolvemento sostible (pp. 531-540). Santiago de Compostela: Universidad de Santiago de Compostela.

Pichs, R. (2007). Situación petrolera mundial y sostenibilidad energética. En J. Mendoza, M. Fernández, y M. Arrastía (Eds.), Educación Enerxética e Desenvolvemento Sostible (pp. 49-68). Santiago de Compostela: Servizo de Publicacións e intercambio Científico, Campus Universitario Sur, Santiago de Compostela.

Postigo, L. (1965). El mundo de la energía. Barcelona: Ramón Sopena.

Romero-Barcos, A. (2006). Progresando hacia una nueva cultura energética. Actuaciones de la ciudad de Barcelona en materia de energética. En J. Mendoza y M. Fernández (Eds.), Educación, Enerxía e Desenvolvemento Sostible (pp. 567-578). Santiago de Compostela: Universidad de Santiago de Compostela.

Sauvé, L. (2004). Una cartografía de corrientes en educación ambiental. En M. Sato e I. Carvalho (Orgs.), A pesquisa em educação ambiental: Cartografias de uma identidade narrativa en formação (pp. 1-22). Porto Alegre: Artmed.

Soberats, Y., González, R., Puentes, V., Díaz-Enríquez, I., Hernández, J., Hernández Álvarez, J. y Puentes, A. (2005). El desarrollo de la conciencia energética y la cultura ecológica en la educación primaria y especial. En M. Fernández, M. Arrastía, J. Fundora y J. Mendoza (Eds.), Educación e Enerxía Propostas sobre a educación Enerxética e o Desenvolvemento Sostible (p. 189). Santiago de Compostela: Servizo de Publicacións e Intercambio Científico, Campus Universitario Sur.

Vilches, A., Gil, D., Toscano, J. y Macias, O. (2007). Agotamiento y destrucción de los recursos naturales. [En línea]. Recuperado de http://www. oei.es/decada/accion23.htm

Weart, S. (2006). El calentamiento global. La historia de un descubrimiento científico. (Trad. J. L. Gil Aristu). Navarra: Laeotoli. 